\title{
Enhanced coupling to microsphere resonances with optical fibers
}

\author{
A. Serpengüzel \\ Department of Physics, Bilkent University, Bilkent, Ankara 06533 Turkey \\ S. Arnold and G. Griffel \\ Microparticle Photophysics and Photonics Laboratories, Polytechnic University, \\ 6 Metrotech Center, Brooklyn, New York 11201 \\ J. A. Lock \\ Department of Physics, Cleveland State University, Cleveland, Ohio 44115
}

Received July 17, 1996

\begin{abstract}
Morphology-dependent resonances (MDR's) of polystyrene microspheres were excited by an optical fiber coupler. For optical elimination of the air-cladding interface at the optical fiber coupler surface, the microsphere was immersed in an index-matching oil. MDR's were observed, even though the relative refractive index between the microsphere and the oil was only 1.09. The observed MDR spectra are in good agreement with the generalized Lorenz-Mie theory and the localization principle. The scattering efficiency into each MDR is estimated as a function of the impact parameter by means of generalized Lorenz-Mie theory. (C) 1997 Optical Society of America [S0740-3224(97)01804-3]

Key words: generalized Lorenz-Mie theory, guided waves, impact parameter, localization, microparticle, morphology-dependent resonances, photonic atom, scattering, whispering-gallery modes.
\end{abstract}

\section{INTRODUCTION}

Because of their unique optical properties, microspheres have enjoyed the attention of the optical spectroscopy community in recent years. ${ }^{1}$ Because of the spherical interface, three electromagnetic and quantumelectrodynamic effects take place in a microsphere. First, the microsphere acts as an optical cavity for specific wavelengths $\lambda$ 's, which satisfy the morphology-dependent resonance (MDR) condition. MDR's can be considered as standing waves, which may be decomposed into two counterpropagating waves traveling around the microsphere rim. Second, the transition cross sections in the microsphere can be larger than bulk transition cross sections, because of the modified density of final electromagnetic states. $^{2}$ In the microsphere, the final states correspond to the microsphere cavity resonances, which are described by MDR's. For a bulk sample, however, the final states are the continuum modes of an infinite system., Third, for plane-wave illumination, the internal intensity is concentrated along the principal diameter near the front and the back surfaces of the microsphere. If the incident plane wave is resonant with a MDR (i.e., on resonance), there will be, in addition, a uniform intensity distribution within the rim of the microsphere in the volume determined by the MDR. ${ }^{5}$ However, if an off-axis Gaussian beam is used at a resonant wavelength, the internal intensity is distributed only within the rim of the microsphere in the volume determined by the MDR and is no longer concentrated near the front and the back surfaces of the microsphere. ${ }^{6}$ Therefore a resonant off-axis
Gaussian beam excites the MDR's of a microsphere more uniformly and more efficiently than does a plane wave. Even edge illumination with a focused beam excites MDR's more efficiently than do plane waves., ${ }^{7,8}$ The offaxis Gaussian beam calculations have recently been realized by means of generalized Lorenz-Mie theory (GLMT). ${ }^{9,10}$ In this paper we report, for the first time to our knowledge, exact formulas for the GLMT scattering cross sections to estimate the efficiency of the coupling (i.e., scattering) into the MDR's.

\section{EXPERIMENTAL SETUP AND OBSERVATIONS}

The first experimental realization of the off-axis Gaussian beam excitation geometry was performed by use of an optical fiber coupler (OFC). ${ }^{11}$ The frequency shift and the linewidth broadening of the MDR's, which are both due to the OFC-microsphere interaction, were also studied. ${ }^{12}$ However, in these OFC-microsphere experiments, the cladding of the fiber was not index matched to the media surrounding the microsphere, so there was an optical interface at the OFC surface. In this paper we report, for the first time to our knowledge, the excitation of the MDR's of a microsphere resting on the surface of an OFC whose surface has been wet with an index-matching oil to eliminate the air-fiber interface. The beam in the optical fiber thereby effectively becomes the equivalent of a Gaussian beam with an infinitely long skirt length. 
Figure 1 is a schematic of our experimental setup. A polystyrene (PS) microsphere with an approximate radius of $12 \mu \mathrm{m}$ and a refractive index of 1.59 is placed on an OFC. The OFC is made from a single-mode optical fiber

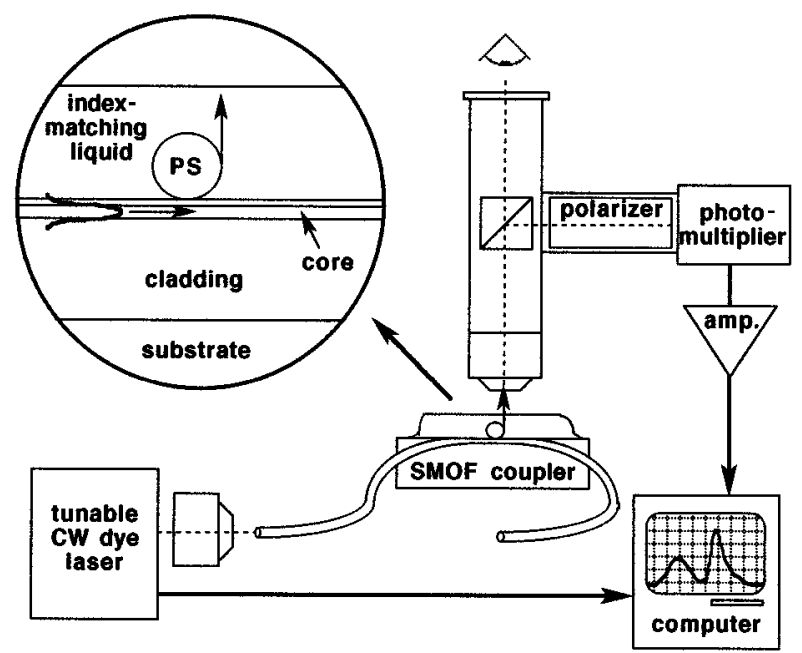

Fig. 1. Schematic of the experimental setup, with inset (top left) depicting the microsphere on the wet surface of the single-mode optical fiber (SMOF) coupler. PS, polystyrene, amp., amplifier.
(SMOF) with a core diameter of $3.8 \mu \mathrm{m}$ (refractive index, 1.462) and a cladding diameter of $125 \mu \mathrm{m}$ (refractive index, 1.457). The cladding below the microsphere is shaved down to $0.7 \mu \mathrm{m}$. The SMOF mode has approximately a Gaussian intensity profile and is doubly degenerate with both horizontal and vertical polarizations. The OFC surface and the microsphere were wet with a few millimeters of index-matching oil (refractive index of 1.456, same as the cladding).

The excitation is provided by a tunable and linearly polarized cw dye laser with optogalvanic calibration and a linewidth of $0.025 \mathrm{~nm}$. The output of the dye laser is coupled to the SMOF with a microscope objective. Although the output of the dye laser was linearly polarized, the output of the SMOF was observed to be elliptically polarized because of the fiber birefringence. Therefore the OFC provides both linear polarizations to the microsphere. The scattering from the microsphere was collected at $90 \pm 5^{\circ}$ with a microscope objective (numerical aperture, 0.17) followed by a polarizer and was detected with a photomultiplier tube.

If one used plane-wave illumination, the image of the microsphere (either on resonance or off resonance) would show three principal glare spots [Fig. 2(a)]. ${ }^{13}$ However, in our case of coupling an external beam from the SMOF,

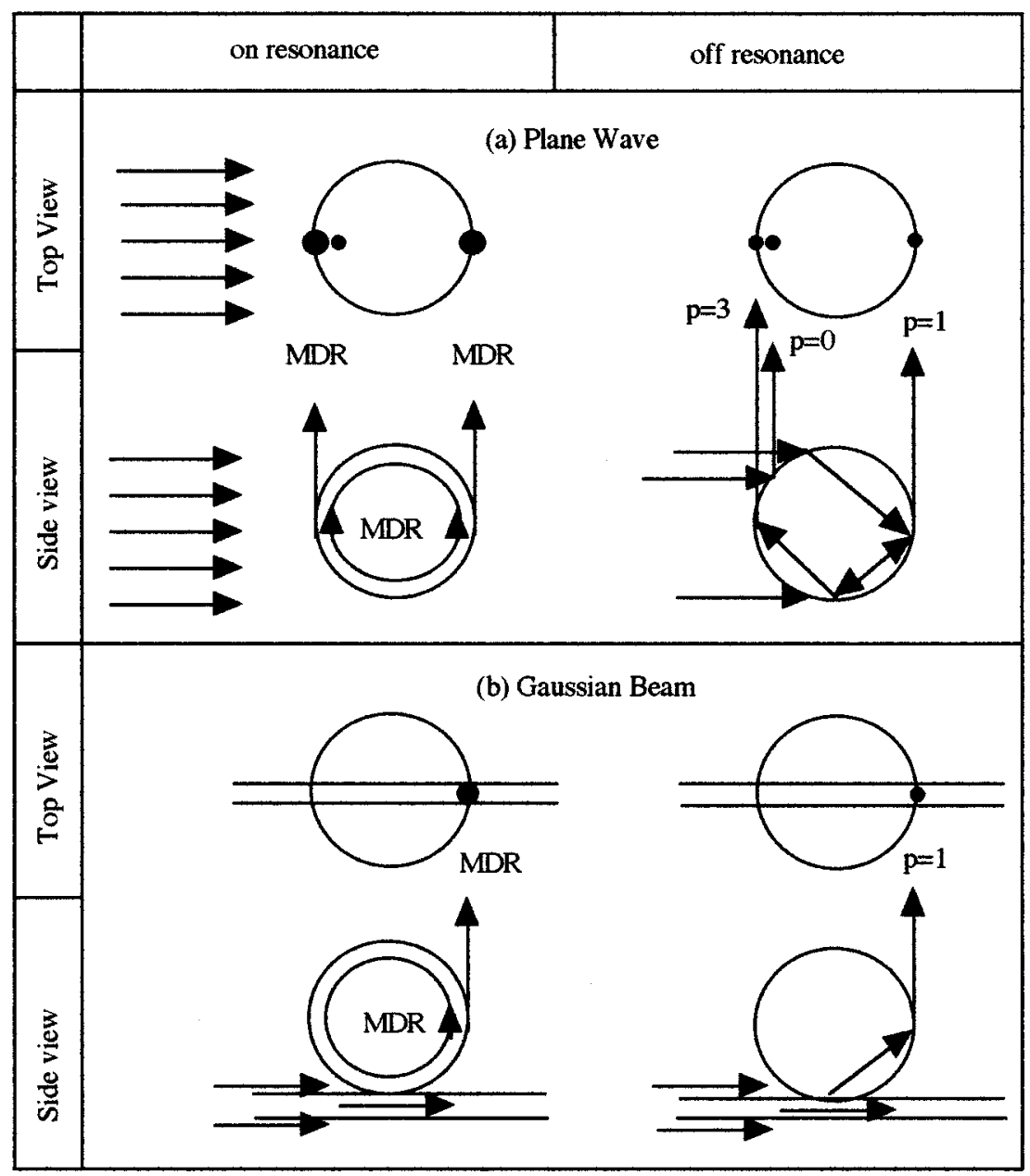

Fig. 2. Schematic of the top and the side views of the microsphere depicting the nonresonant $(p=0,1,3)$ glare spots $(\bullet)$ and MDR glare spots ( ) when excited (a) by a plane wave or (b) with the OFC (Gaussian beam) for both on- and off-resonance conditions. 


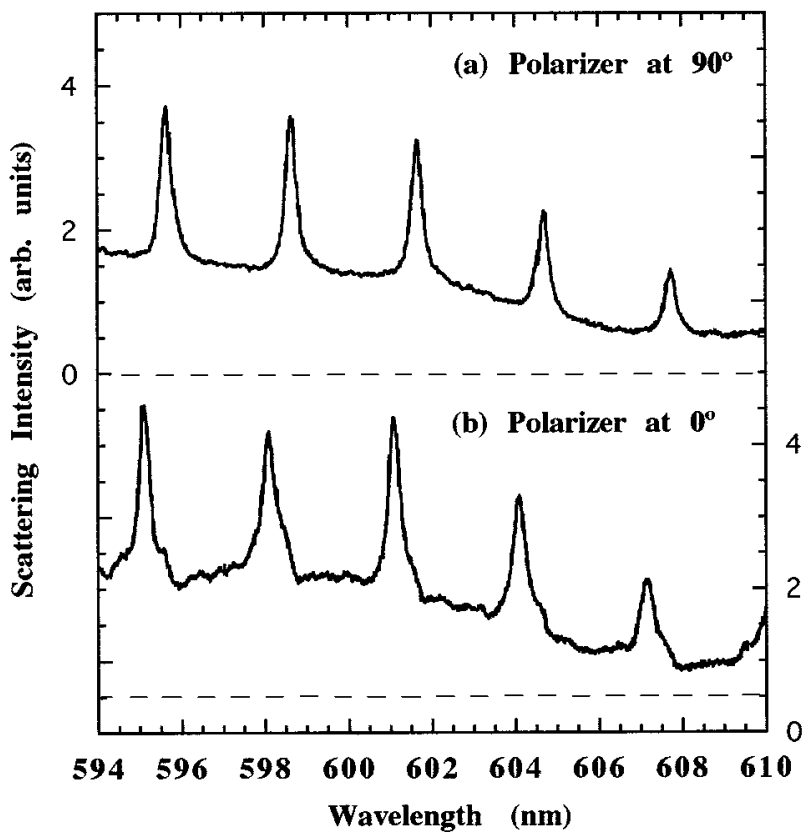

Fig. 3. Experimental results obtained with the polarizer at (a) $90^{\circ}$ and (b) $0^{\circ}$ to the SMOF.

we observe only one glare spot on the far side of the microsphere [Fig. 2(b)]. In contrast to the non-indexmatched case, this far-side glare spot is observed for all the laser wavelengths, even when the incident wavelength is not on a MDR (i.e., off resonance). However, when the incident light is on resonance, the far-side glare spot intensity is enhanced by a factor of $\sim 2$. Apparently, the standing-wave pattern that is set up by a plane-wave excitation of a MDR, with its counterpropagating traveling waves [Fig. 2(a)], is replaced with a single counterclockwise traveling wave in the Gaussian beam excitation [Fig. 2(b)]. Also, in the Gaussian beam excitation [Fig. 2 (b)], the off-resonance glare spots are due only to refraction (i.e., $p=1$ rays), whereas for a plane-wave illumination the off-resonance glare spots are due to both refraction (i.e., $p=1,3$ rays) and specular reflection (i.e., $p$ $=0$ rays).

Figure 3 shows the scattering spectra at a scattering angle of $90 \pm 5^{\circ}$ from the microsphere; these were obtained through a polarizer with its polarization axis at $90^{\circ}$ (a) and (b) $0^{\circ}$ to the SMOF. The MDR's that are present in Fig. 3(a) are missing when compared with Fig. $3(\mathrm{~b})$, a result that attests to the polarization dependence of the MDR's. From the polarizer orientation, we can deduce that the MDR's that are present in Fig. 3(a) are the transverse-electric (TE) type and that the MDR's that are present in Fig. 3(b) are the transverse-magnetic (TM) type. Both spectra have been normalized by the laser intensity spectrum, which decreases continually with increasing wavelength. To rule out the ellipticity of the microparticle, we also observed the plane-wave scattering spectra (not shown) at a scattering angle of $45^{\circ}$ from the microparticle and confirmed that the microparticle was indeed spherical, since the MDR's corresponding to different great-circle trajectories occurred at the same wavelength. ${ }^{14}$
Comparing the spectra shown in Fig. 3 with the scattering spectra obtained with a plane wave, we noticed several features: (1) There is a large background intensity, which cannot be explained by the light scattering that results from OFC surface imperfections; and (2) The MDR's have nearly Lorentzian line shapes as opposed to the Fano line shapes ${ }^{15}$ of the plane-wave scattering spectra.

\section{THEORY OF OFF-AXIS MORPHOLOGY- DEPENDENT RESONANCE EXCITATION}

Most of the prominent features of these scattering spectra are described by the interaction of the microsphere with an external beam having a Gaussian intensity profile and propagating at an impact parameter $b$, which is slightly greater than the microsphere radius a. Since the excitation by such a beam occurs beyond the edge of the microsphere, the light scattering can be calculated by (i) removal of the partial waves with angular-momentum quantum numbers $n$ less than the size parameter $(x$ $=2 \pi \mathbf{a} / \lambda$ ) from the conventional Lorenz-Mie (planewave excitation) infinite series, and (ii) application of GLMT to parameterize the incident-beam profile. This removal of partial waves is justified by the localization principle,${ }^{16}$ which associates a light ray having an impact parameter $b$ with a partial wave with mode number $n$. This relationship can be expressed as follows:

$$
b=\left(n+\frac{1}{2}\right) \frac{\mathbf{a}}{x}
$$

Plane-wave Lorenz-Mie theory restricts the angularmomentum quantum number $n$ range of the light rays, passing by the microsphere surface but yet interacting with it, to be $N x \geqslant n \geqslant x$, where $N$ is the relative refractive index of the microsphere with respect to the outside medium. This condition, together with the localization principle, restricts the impact parameter $b$ range to be $N \mathbf{a} \geqslant b \geqslant \mathbf{a}$. Therefore only the light rays within the impact parameter range of $N \mathbf{a} \geqslant b \geqslant \mathbf{a}$ can couple to the MDR's of the microsphere.

To check the validity of this approach and to simulate our experimental results, we used the GLMT computation algorithm of Ref. 17 (Figs. 4 and 5), which can be applied to on- and off-axis focused Gaussian beam excitation geometries. In GLMT, the plane-wave partial-wave expansion coefficients ( $a_{n}$ for TM or $b_{n}$ for TE resonances) are replaced by the partial-wave expansion coefficients $\left(a_{n m}\right.$ for TM coefficients or $b_{n m}$ for TE coefficients). For example, for TE contributions, $b_{n m}=b_{n} B_{n}{ }^{m}$, where

$$
B_{n}{ }^{m}=\frac{\mathbf{a}^{2}}{n(n+1) \psi_{n}(x)} \int_{0}^{4 \pi} \mathrm{d} \Omega H_{r}(\Omega) Y_{n m} *(\Omega),
$$

with $\psi_{n}(x)$ being a Ricatti-Bessel function; $\Omega$, the solid angle; $H_{r}(\Omega)$, the radial component of the incident magnetic field evaluated on the microsphere surface $(r=\mathbf{a})$; and $Y_{n m} *(\Omega)$, a spherical harmonic function. In other words, $B_{n}{ }^{m}$ describe the angular overlap of the excitation field and the spherical harmonics.

For our external off-axis Gaussian beam excitation geometry and as seen in Ref. $17, B_{n}{ }^{m}$ become significant as 


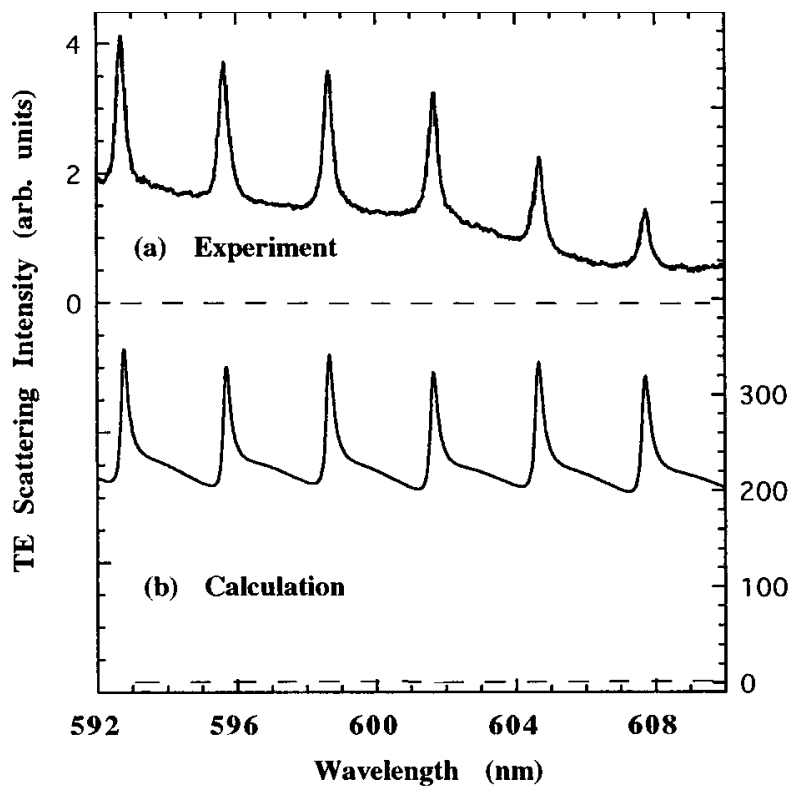

Fig. 4. Scattering intensity of (a) experimental and (b) calculated TE-polarized spectrum.

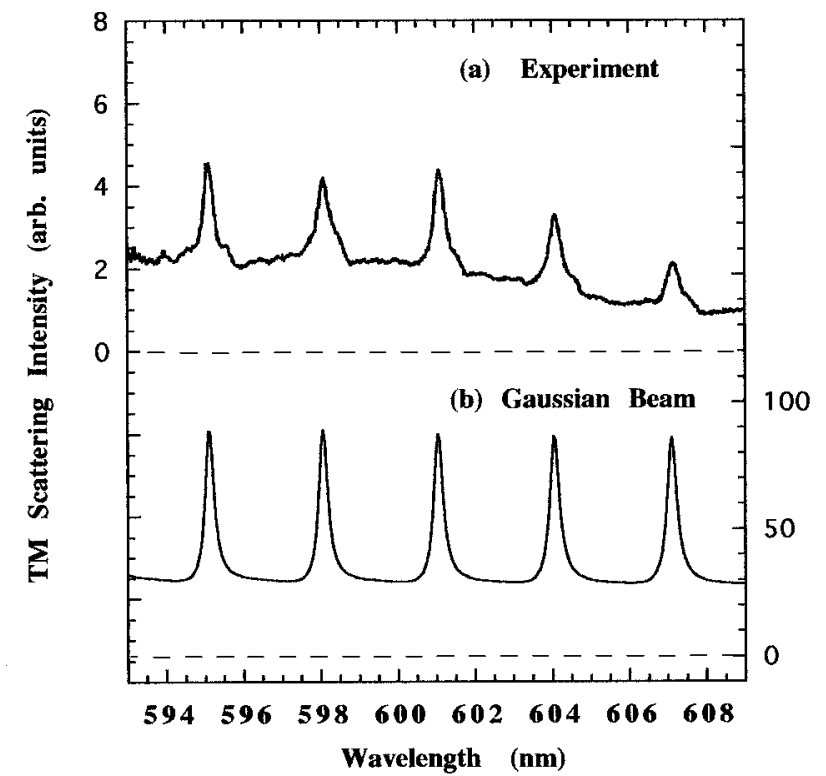

Fig. 5. Scattering intensity of (a) experimental and (b) calculated TM-polarized spectrum.

$n$ exceeds $x$. As $n$ is increased further, $B_{n}{ }^{m}$ goes through a series of resonances (i.e., MDR's), with none being seen beyond $n=N x$. The background in the spectra of Figs. 4 (b) and 5(b) is due to the refraction for nonresonant partial waves with $n<x$. For a plane-wave excitation, $B_{n}{ }^{m}$ would have been significant for all $n$.

For the calculations [Figs. 4(b) and 5(b)], we used a Gaussian beam (with an infinite skirt length and a beam waist with a half-width $\omega_{0}=2.176 \mu \mathrm{m}$ ) propagating at an impact parameter of $14.94 \mu \mathrm{m}$ from a microsphere (with $\mathbf{a}=12.34 \mu \mathrm{m}$ and a relative refractive index of 1.09). The calculation of the scattered intensity is averaged over the scattering angle from $90 \pm 5^{\circ}$. The results of this calculation [Figs. 4(b) and 5(b)] are compelling. The theoretical MDR's for an off-axis incidence bear a good relationship to the experimental data [Figs. 4(a) and 5(a)] and appear to correspond to first-order MDR's with theoretical quality factors Q's of approximately 2000. The mode numbers $n$ for the MDR's are within the range of $n=194-198$.

By using the localization principle, we can estimate where the beam should have been placed for maximal excitation of these MDR's with $n=194-198$. We obtain $b=n / k=12.9 \mu \mathrm{m}$ by using Eq. (1), which is smaller than our experimental value of $b=14.94 \mu \mathrm{m}$. Therefore we are not coupling to these MDR's with $n=194-198$ with maximal efficiency.

The coupling (scattering) efficiency can be estimated by calculation of the power coupled (scattered) into the partial-wave modes. The power coupled (scattered) into a mode can be calculated by use of the scattering cross section $\sigma$. The power coupled into a mode can be expressed as the ratio of the partial-wave scattering cross section $\sigma_{\text {mode }}$ to the total cross section $\sigma_{\text {total }}$ multiplied by the incident beam power $P_{i}$ or, equivalently, $\sigma_{\text {mode }}$ multiplied by the average incident beam intensity $\left\langle I_{i}\right\rangle$. The power leaking (coupling out) from a mode is proportional to the energy $\epsilon$ stored in the mode divided by the time constant $\tau$ of that mode. Hence the total power going into a mode can be expressed as

$$
\frac{\mathrm{d} \epsilon}{\mathrm{d} t}=\frac{\sigma_{\text {mode }}}{\sigma_{\text {total }}} P_{i}-\frac{\epsilon}{\tau}=\left\langle\sigma_{\text {mode }}\right\rangle P_{i}-\frac{\epsilon}{\tau}=\sigma_{\text {mode }}\left\langle I_{i}\right\rangle-\frac{\epsilon}{\tau},
$$

where we have defined $\left\langle\sigma_{\text {mode }}\right\rangle$ as the scattering (or power coupling) efficiency into a mode. Then the steady-state energy $\epsilon_{0}$ in the mode can be expressed as

$$
\epsilon_{0}=\frac{\sigma_{\text {mode }}}{\sigma_{\text {total }}} P_{i} \tau=\left\langle\sigma_{\text {mode }}\right\rangle P_{i} \tau=\sigma_{\text {mode }}\left\langle I_{i}\right\rangle \tau .
$$

For a plane wave, the total cross section $\sigma_{\text {total }}$ is $2 \pi \mathbf{a}^{2}$. A Gaussian beam with the same intensity as the plane wave, however, has a total cross section of $\pi \omega_{0}^{2} / 2$. Therefore an off-axis Gaussian beam will couple more efficiently into a mode if it happens to be propagating at the impact parameter $b$ corresponding to that mode. One can estimate the scattering efficiency for a Gaussian beam $\left(\left\langle\sigma_{\text {mode }}\right\rangle_{\text {Gaussian }}\right)$ by following this geometrical point of view. Figure 6 is a sketch of the transverse view of the Gaussian beam with area $\pi \omega_{0}^{2}$ and the cross-sectional mode area $(2 \pi b d b)$. The mode thickness $d b$ can be estimated from the localization principle $\left(b_{n}=n / k\right)$ to be $d b=1 / k$. Only the part of the Gaussian beam that overlaps with the ribbon contributes to the coupling to a specific mode. This area is $\approx \sqrt{2} \omega_{0} d b=\sqrt{2} \omega_{0} / k$ $=\omega_{0} \lambda / \sqrt{2} \pi$. We then obtain $\left\langle\sigma_{\text {mode }}\right\rangle_{\text {Gaussian }}$ $\approx \sqrt{2} \lambda / \pi^{2} \omega_{0}=0.03=3 \%$ (for our experimental parameters and assuming that our beam was propagating at the appropriate impact parameter). For a plane-wave excitation, however, $\left\langle\sigma_{\text {mode }}\right\rangle_{\text {plane wave }} \approx \lambda / 2 \pi \mathbf{a}=1 / x=0.005$ $=0.5 \%$ (for our experimental parameters).

For a better estimate of the power scattered (coupled) into a mode, we can use Lorenz-Mie theory. For a planewave illumination, the scattering cross section for a TMpolarized partial wave $\sigma_{a n}$ is ${ }^{18}$ 


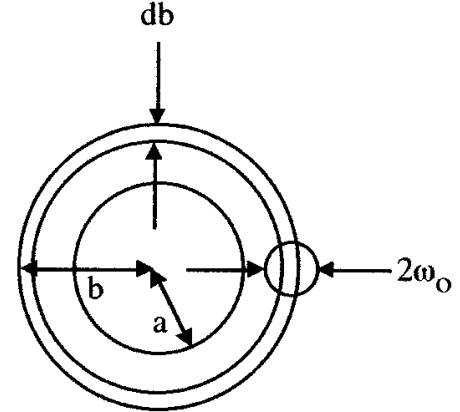

Fig. 6. Transverse view of the ratio of the Gaussian beam area $\pi \omega_{0}{ }^{2}$ and the cross-sectional area $(2 \pi b d b)$ corresponding to a mode. The microsphere radius is a.

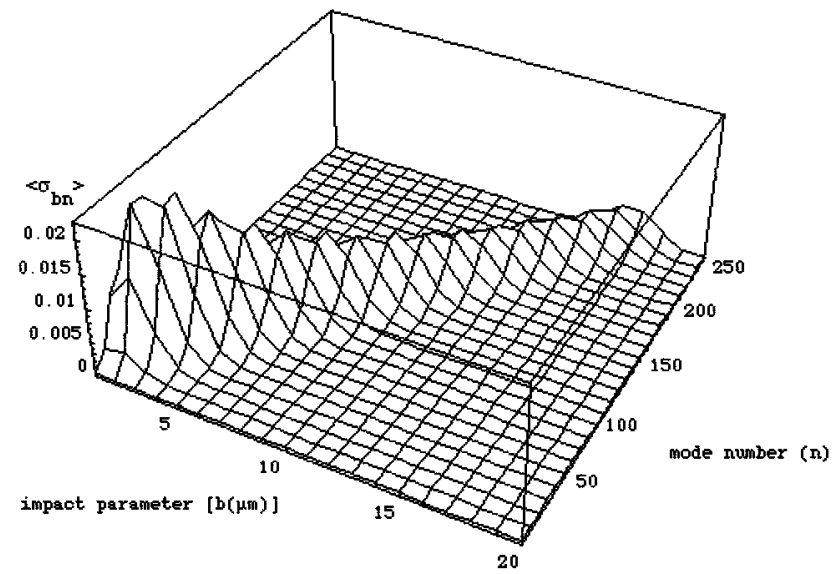

Fig. 7. Scattering efficiency $\left\langle\sigma_{b n}\right\rangle$ for TE modes for a Gaussian beam illuminating a microsphere with our experimental parameters plotted as a function of the impact parameter $b$ and the angular-momentum quantum (mode) number $n$.

$$
\sigma_{a n}=\frac{2 \pi}{k^{2}}\left|a_{n}\right|^{2}(2 n+1),
$$

where $a_{n}$ is the scattered-electric-field coefficient and $n$ is the angular-momentum quantum (mode) number. Similarly, the scattering cross section for a TE-polarized partial wave $\sigma_{b n}$ is

$$
\sigma_{b n}=\frac{2 \pi}{k^{2}}\left|b_{n}\right|^{2}(2 n+1),
$$

where $b_{n}$ is the scattered-electric-field coefficient. Hence the total scattering cross section $\sigma_{\text {total }}$ is

$$
\begin{aligned}
\sigma_{\text {total }}= & \sum_{n=1}^{\infty} \sigma_{a n}+\sigma_{b n} \\
= & \frac{2 \pi}{k^{2}} \sum_{n=1}^{\infty}\left(\left|a_{n}\right|^{2}+\left|b_{n}\right|^{2}\right) \\
& \times(2 n+1)=2 \pi \mathbf{a}^{2} .
\end{aligned}
$$

For the more general case of an off-axis Gaussian beam illumination, we calculated the scattering cross section for a TM-polarized partial wave $\sigma_{a n}$ to be

$$
\sigma_{a n}=\frac{2 \pi}{k^{2}}\left|a_{n}\right|^{2}\left[\frac{2 n+1}{2 n(n+1)}\right] \sum_{m=-n}^{n}\left|A_{n}^{m}\right|^{2} \frac{(n+|m|) !}{(n-|m|) !},
$$

where $A_{n}{ }^{m}$ is the generalized Lorenz-Mie coefficient of the scattered electric field and $m$ is the azimuthal quantum mode number. Similarly, we have calculated the scattering cross section for a TE-polarized partial wave $\sigma_{b n}$ to be

$$
\sigma_{b n}=\frac{2 \pi}{k^{2}}\left|b_{n}\right|^{2}\left[\frac{2 n+1}{2 n(n+1)}\right] \sum_{m=-n}^{n}\left|B_{n}{ }^{m}\right|^{2} \frac{(n+|m|) !}{(n-|m|) !},
$$

where $B_{n}{ }^{m}$ is the generalized Lorenz-Mie coefficient of the scattered electric field.

For the more symmetric on-axis Gaussian beam (i.e., with $m= \pm 1$ ), the expressions for the TM-polarized partial-wave scattering cross section $\sigma_{a n}$ reduce to

$$
\sigma_{a n}=\frac{2 \pi}{k^{2}}\left|a_{n}\right|^{2}(2 n+1)\left|A_{n}^{ \pm 1}\right|
$$

and the expressions for the TE-polarized partial-wave scattering cross section $\sigma_{b n}$ reduce to

$$
\sigma_{b n}=\frac{2 \pi}{k^{2}}\left|b_{n}\right|^{2}(2 n+1)\left|B_{n}{ }^{ \pm 1}\right| .
$$

All these scattering cross sections (Eqs. 7 and 8) reduce to the plane-wave scattering cross sections [Eqs. (5)], for a plane-wave excitation (with $\left|A_{n}{ }^{ \pm 1}\right|=\left|B_{n}{ }^{ \pm 1}\right|=1$ ).

Using Eqs. (7) for an off-axis Gaussian beam illuminating our microsphere, we calculated (Fig. 7) the scattering efficiency $\left(\left\langle\sigma_{b n}\right\rangle=2 \sigma_{b n} / \pi \omega_{0}^{2}\right)$ for the TE modes as a function of the impact parameter $b$ and the angularmomentum quantum (mode) number $n$. Different mode numbers are excited at different impact parameters. For our modes with $n=194-198$, in accordance with the localization principle, the maximum of the scattering efficiency occurs at an impact parameter of $12.9 \mu \mathrm{m}$. At our impact parameter of $14.94 \mu \mathrm{m}$, the scattering efficiency is less than $1 \%$. To couple most efficiently (i.e., $1 \%$ is a factor of 3 smaller than our geometric estimate of $3 \%$ ) to our modes with $n=194-198$, we would have had to excite the microsphere at an impact parameter of $12.9 \mu \mathrm{m}$.

However, efficient coupling is not sufficient for maximum energy storage in the microsphere. As can be seen from Eq. 4, the total energy that can be stored in the mode is proportional to the lifetime $\tau$ of that mode, which can further be enhanced with the use of a higher refractive-index contrast between the PS microsphere and its surrounding medium, e.g., $N=1.09$ (indexmatching liquid) $\rightarrow 1.18$ (water). Although the water results are more difficult to simulate because of the refractive-index step at the cladding-water interface and because the excitation electric field is evanescent rather than Gaussian, it is possible to have modes with higher measured $Q$ 's of $\approx 24,000$. Figure 8 shows the experimental elastic scattering intensity of the TE-polarized spectrum for a PS microsphere in water $(N=1.18)$. These TE resonances are suppressed when the polarizer axis is parallel to the SMOF (not shown). A noteworthy 


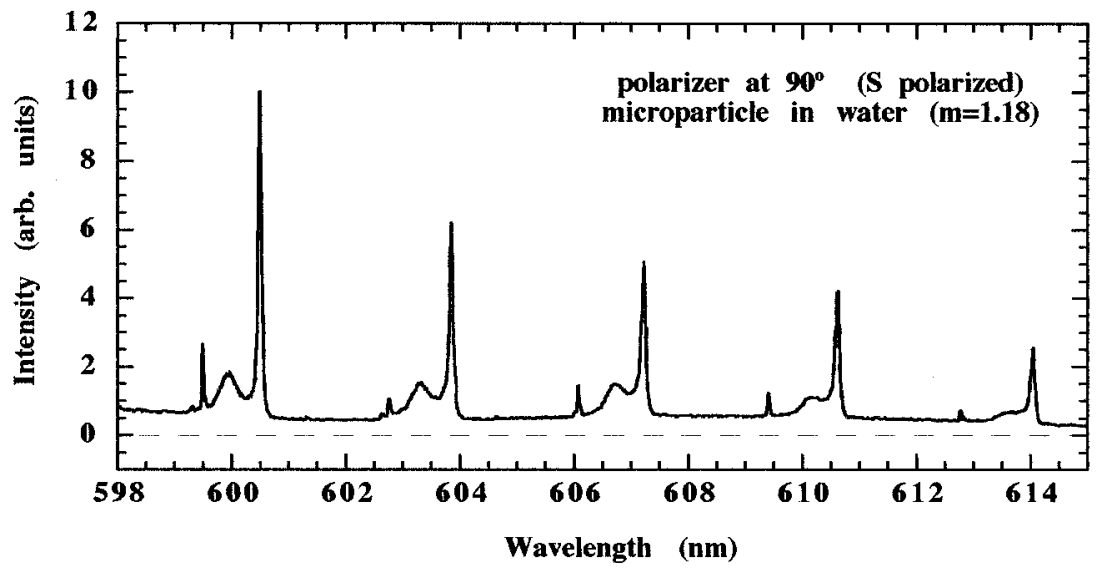

Fig. 8. Scattering intensity of the experimental TE-polarized spectrum for a PS microsphere in water $(m=1.18)$.

feature of the spectra shown in Fig. 8 is that MDR's with linewidths as narrow as $0.04 \mathrm{~nm}$ were observed. Since our dye laser has a linewidth of $0.025 \mathrm{~nm}$, the measured MDR linewidths are clearly limited by the convolution of our dye laser linewidth.

\section{CONCLUSION}

In conclusion, the microsphere-optical fiber system with the index-matching geometry has proved to be very useful in the verification of the generalized Lorenz-Mie theory and the localization principle. The microsphere-optical fiber system also shows promise as a possible building block for photonic memories ${ }^{19}$ and can be used as an external-cavity feedback system to line narrow a broader light source such as a diode laser..$^{20,21}$

\section{ACKNOWLEDGMENTS}

We are grateful for research support from the U.S. Air Force Office of Scientific Research (grant F49620-940195) and the Scientific and Technical Research Council of Turkey (TUBITAK grant TBAG-1368).

\section{REFERENCES}

1. P. W. Barber and R. K. Chang, eds., Optical Effects Associated with Small Particles (World Scientific, Singapore, 1988), pp. 3-61.

2. E. M. Purcell, "Spontaneous emission probabilities at radio frequencies," Phys. Rev. 69, 681 (1946).

3. S. C. Ching, H. M. Lai, and K. Young, "Dielectric microspheres as optical cavities: thermal spectrum and density of states,” J. Opt. Soc. Am. B 4, 1995 (1987).

4. S. C. Ching, H. M. Lai, and K. Young, "Dielectric microspheres as optical cavities: Einstein $A$ and $B$ coefficients and level shift," J. Opt. Soc. Am. B 4, 2004 (1987).

5. D. S. Benincasa, P. W. Barber, J.-Z. Zhang, W.-F. Hsieh, and R. K. Chang, "Spatial distribution of the internal and near-field intensities of large cylindrical and spherical scatterers," Appl. Opt. 26, 1348 (1987).

6. E. E. M. Khaled, S. C. Hill, and P. W. Barber, "Internal electric energy in a spherical particle illuminated with a plane wave or off-axis Gaussian beam," Appl. Opt. 33, 524 (1994).

7. J.-Z. Zhang, D. H. Leach, and R. K. Chang, "Photon lifetime within a droplet: temporal determination of elastic and stimulated Raman scattering," Opt. Lett. 13, 270 (1988).

8. J. P. Barton, D. R. Alexander, and S. A. Schaub, "Internal and near-surface electromagnetic fields for a spherical particle irradiated by a focused laser beam," J. Appl. Phys. 64, 1632 (1988).

9. J. A. Lock and G. Gouesbet, "Rigorous justification of the localized approximation to the beam shape coefficients in generalized Lorenz-Mie theory. I. On-axis beams," J. Opt. Soc. Am. A 11, 2503 (1994).

10. G. Gouesbet and J. A. Lock, "Rigorous justification of the localized approximation to the beam shape coefficients in generalized Lorenz-Mie theory. II. Off-axis beams," J. Opt. Soc. Am. A 11, 2516 (1994).

11. A. Serpengüzel, S. Arnold, and G. Griffel, "Excitation of resonances of microspheres on an optical fiber," Opt. Lett. 20, 654 (1995)

12. N. Dubreuil, J. C. Knight, D. K. Leventhal, V. Sandoghar, J. Hare, and V. Lefèvre, "Eroded monomode optical fiber for whispering-gallery mode excitation in fused-silica microspheres," Opt. Lett. 20, 813 (1995)

13. S. Arnold, S. Holler, J. H. Li, A. Serpengüzel, W. F. Auffermann, and S. C. Hill, "Aerosol particle microphotography and glare spot absorption spectroscopy," Opt. Lett. 20, 773 (1995).

14. G. Chen, Md. M. Mazumder, Y. R. Chemla, A. Serpengüzel, R. K. Chang, and S. C. Hill, "Wavelength variation of laser emission along the entire rim of slightly deformed microdroplets," Opt. Lett. 18, 1993 (1993).

15. P. W. Barber and R. K. Chang, eds., Optical Effects Associated with Small Particles (World Scientific, Singapore, 1988), p. 20.

16. H. C. van de Hulst, Light Scattering by Small Particles (Dover, New York, 1981), p. 208.

17. J. A. Lock, "An improved Gaussian beam scattering algorithm," Appl. Opt. 34, 559 (1995).

18. C. F. Bohren and D. R. Huffman, Absorption and Scattering of Light by Small Particles (Wiley, New York, 1983), p. 103.

19. S. Arnold, C. T. Liu, W. B. Whitten, and J. M. Ramsey, "Room-temperature microparticle-based persistent spectral hole burning memory," Opt. Lett. 16, 420 (1991).

20. G. Griffel, A. Serpengüzel, and S. Arnold, "Quenching of semiconductor lasers linewidth by detuned loading using spherical cavities morphology dependent resonances," in Proceedings of IEEE Frequency Control Conference (Institute of Electrical and Electronics Engineers, New York, 1995), pp. 495-497.

21. G. Griffel, S. Arnold, D. Taskent, A. Serpengüzel, J. Connolly, and N. Morris, "Morphology-dependent resonances of a microsphere-optical fiber system," Opt. Lett. 21, 695 (1996). 